\title{
Cellular characterisation of Candida tropicalis presenting fluconazole-related trailing growth
}

\author{
Marcos Dornelas-Ribeiro, ${ }^{1,2,3,4}$, Eliane Olmo Pinheiro ${ }^{3}$, Carolina Guerra ${ }^{5}$, Lys Adriana Braga-Silva ${ }^{6,7}$, \\ Silvia Maia Faria de Carvalho², André Luis Souza dos Santos ${ }^{6,7}$, \\ Sonia Rozental ${ }^{5}$, Sergio Eduardo Longo Fracalanzza ${ }^{4}{ }^{+}$
}

\author{
${ }^{1}$ Laboratório de Bacteriologia/Micologia, Instituto de Biologia do Exército, Rio de Janeiro, RJ, Brasil \\ ${ }^{2}$ Laboratório de Bacteriologia/Micologia, Instituto Estadual de Hematologia, Rio de Janeiro, RJ, Brasil \\ ${ }^{3}$ Laboratório de Bacteriologia/Micologia, Hospital Adventista Silvestre, Rio de Janeiro, RJ, Brasil \\ ${ }^{4}$ Laboratório de Bacteriologia Médica, Departamento de Microbiologia Médica ${ }^{6}$ Laboratório de Estudos Integrados \\ em Bioquímica Microbiana, Departamento de Microbiologia Geral, Instituto de Microbiologia Paulo de Góes \\ ${ }^{5}$ Laboratório de Biologia Celular de Fungos, Instituto de Biofísica Carlos Chagas Filho, ’’Programa de Pós-Graduação em Bioquímica, \\ Instituto de Química, Centro de Tecnologia, Universidade Federal do Rio de Janeiro, Rio de Janeiro, RJ, Brasil
}

We assessed fluconazole susceptibility in 52 Candida tropicalis clinical strains using seven antifungal susceptibility methods, including broth microdilution (BMD) [standard M27 A3 (with neutral and acid pH), ATB Fungus 3 , Vitek 2 system and flow cytometric analysis] and agar-based methods (disk diffusion and E-test). Trailing growth, detection of cell-associated secreted aspartic proteases (Saps) and morphological and ultrastructural traits of these clinical strains were also examined. The ranges of fluconazole 24 h-minimum inhibitory concentration (MIC) values were similar among all methods. The essential agreement among the methods used for MIC determinations was excellent and all methods categorised all strains as susceptible, except for one strain that showed a minor error. The presence of the trailing effect was assessed by six methods. Trailing positivity was observed for $86.5-100 \%$ of the strains. The exception was the BMD-Ac method where trailing growth was not observed. Morphological and ultrastructural alterations were detected in $\mathrm{C}$. tropicalis trailing cells, including mitochondrial swelling and cell walls with irregular shapes. We tested the production of Saps in $13 \mathrm{C}$. tropicalis strains expressing trailing growth through flow cytometry. Our results showed that all of the C. tropicalis strains up-regulated surface Sap expression after $24 \mathrm{~h}$ or $48 \mathrm{~h}$ of exposure to fluconazole, which was not observed in untreated yeast strains. We concluded that $\mathrm{C}$. tropicalis strains expressing trailing growth presented some particular features on both biological and ultrastructural levels.

Key words: Candida tropicalis - antifungal susceptibility test - trailing growth biological and ultrastructural alterations - aspartic protease production

The increased incidence of systemic mycoses caused by Candida species in hospitalised patients is an important cause of morbidity and mortality worldwide, especially in critically ill patients (Warnock 2007, Mimica et al. 2009, Ruan \& Hsueh 2009). In this context, immunocompromised individuals and patients subjected to broad-spectrum antibiotics, using prostheses and access devices and experiencing prolonged stays in intensive care units are predisposed to Candida infections (Sandven 2000, Pfaller \& Diekema 2004, 2007, Fridkin et al. 2006). More than 200 species of Candida have been described, but approximately $90 \%$ of human invasive fungal infections are caused by only five species: Candida albicans, Candida tropicalis, Candida parapsilosis, Candida glabrata and Candida krusei (Pfaller \& Diekema 2004).

C. tropicalis is the second or third most abundant species of Candida non-albicans. This organism has been isolated in patients with cancer, particularly leukaemia, more

+ Corresponding author: fracalanzza@micro.ufrj.br

Received 7 April 2011

Accepted 16 August 2011 frequently (Cantón et al. 2001, Colombo \& Guimarães 2003). C. tropicalis is more invasive in relation to C. albicans and it is estimated that $50-60 \%$ of patients with predisposing conditions develop invasive candidiasis when they are colonised by this species (Cantón et al. 2001).

When tested against the azoles, some species of the genus Candida, mainly C. albicans and C. tropicalis, may express a trailing phenomenon, which is defined as a susceptible minimum inhibitory concentration (MIC) after $24 \mathrm{~h}$ of incubation with azole antifungal agents, but a resistant MIC after $48 \mathrm{~h}$ of exposure (Revankar et al. 1998, Arthington-Skaggs et al. 2000). As a consequence, the trailing phenotype results in a misinterpretation of the susceptibility profile for these drugs, reflecting a possible treatment failure. With this propose in mind, some studies have examined the methods to minimise the trailing effect and changes in the National Committee on Clinical Laboratory Standards/Clinical and Laboratory Standards Institute (NCCLS/CLSI) methodology, such as a more acid $\mathrm{pH}$, addition of glucose to the medium and a cut-off criterion for the reading time have been proposed (Anaissie et al. 1996, Rodriguez-Tudela et al. 1996, Tornatore et al. 1997, Marr et al. 1999). Mechanisms have been proposed to explain the trailing effect, including the altered regulation of genes mediating resistance and virulence (Lee et al. 2004). 
Pathogenic species of Candida spp produce many potential virulence factors and among them, the secreted aspartic proteases (Saps) have attracted the attention of many investigators (Naglik et al. 2003). A direct correlation exists between the virulence of these yeast strains and the secretion of Saps; consequently, these enzymes are becoming attractive targets for therapeutic drug design (Braga-Silva \& Santos 2011). Ten different Saps, encoded by the $S A P$ gene family, were described in $C$. albicans (Naglik et al. 2003) and four SAP genes were found in C. tropicalis (Zaugg et al. 2001). Moreover, immunological studies suggest the existence of common and specific antigenic domains among the Saps of these two clinically important Candida species (Odds 1985). At least in C. albicans, Saps participate in numerous facets of basic biological processes and directly participate in several interactions between fungal cells and host structures (Naglik et al. 2003).

In the present study we have aimed to characterise the trailing growth of $52 \mathrm{C}$. tropicalis clinical isolates using different antifungal susceptibility methods. In addition, the expression of surface Saps and the morphological/ultrastructural alterations in these strains were also reported.

\section{SUBJECTS, MATERIALS AND METHODS}

Patients and strains - Of a total of 52 C. tropicalis clinical strains isolated from patients in three medical centres in the city of Rio de Janeiro, Brazil, 29 (55.7\%) were from blood, eight $(15.3 \%)$ from urine, five $(9 \%)$ from faeces, three $(6 \%)$ from skin material, three $(6 \%)$ from secretion, two $(4 \%)$ from sterile fluid, one $(2 \%)$ from bronchoalveolar lavage and one $(2 \%)$ from lymph node aspirate. Strains were isolated and identified by conventional methods and the identification was confirmed using the $\operatorname{Vitek}^{\circledR} 2$ system (bioMérieux, Inc, France). Isolates were stored at $-70^{\circ} \mathrm{C}$ in Sabouraud dextrose broth with $20 \%$ glycerol and at room temperature in sterile distilled water (Lee et al. 2004).

Susceptibility testing - Testing of the susceptibility of C. tropicalis isolates to fluconazole and trailing growth characterisation were performed using seven methods. Three of these methods were standardised by CLSI [broth microdilution (BMD) tests in accordance with M27 A3 guideline at neutral pH (BMD-N) and acid pH (BMD-Ac)] (CLSI 2008) and NCCLS [disk diffusion test (DT) according to the M44-A guideline] (NCCLS 2004). The other four methods included commercial systems $\left[\mathrm{ATB}^{\circledR}\right.$ Fungus 3 (ATBF3) (bioMérieux, Inc, France), Vitek 2 and E-test method (AB Biodisk, Solna, Sweden)] and the flow cytometric analysis (Ramani et al. 1997, Ramani \& Chaturvedi 2000).

Inoculums - A suspension of five colonies, from up to $48 \mathrm{~h}$-old cultures grown at $35^{\circ} \mathrm{C}$ on Sabouraud dextrose agar, was prepared in $5 \mathrm{~mL}$ of sterile saline $(0.85 \% \mathrm{NaCl})$. The cell density was standardised with a spectrophotometer, where diluent was added to the yeast suspension until it matched the value of a 0.5 McFarland standard at a $530 \mathrm{~nm}$ wavelength $\left(1 \times 10^{6}-5 \times 10^{6}\right.$ yeast cells $\left./ \mathrm{mL}\right)$. This standardised inoculum was used in the following methods:
BMD-N and BMD-Ac, disk diffusion, E-test and flow cytometric analysis. For the ATBF3 and Vitek 2 methods, the inoculum was adjusted to $3-6 \times 10^{6}$ yeasts $/ \mathrm{mL}$.

CLSI reference procedure (M27-A3 guideline): $B M D-N$ and $B M D-A c$ - Serial two-fold dilutions of fluconazole were prepared in Roswell Park Memorial Institute (RPMI)-1640 medium with L-glutamine and without bicarbonate (Sigma Chemical Co). The final concentrations of fluconazole ranged from $0.25-256 \mu \mathrm{g} /$ $\mathrm{mL}$ and the $\mathrm{pH}$ value was adjusted to neutral (with a $165 \mathrm{mM}$ morpholinepropanesulfonic acid buffer) or acid (pH 5.0, with $\mathrm{HCl}$ ). A $100 \mu \mathrm{L}$ aliquot of each drug dilution was dispensed into 96-well flat-bottom microdilution trays that were sealed and stored frozen at $-70^{\circ} \mathrm{C}$ until use. The standardised suspension was diluted 1:50 and then 1:20 in RPMI with L-glutamine and without sodium bicarbonate, resulting in $1 \times 10^{3}-5 \times 10^{3}$ cells $/ \mathrm{mL}$. A $100 \mu \mathrm{L}$ aliquot of the final suspension was put into each well of the microdilution plate and the plates were incubated at $35^{\circ} \mathrm{C}$ for $48 \mathrm{~h}$. As a viability, purity and concentration of inoculum control, aliquots $(10 \mu \mathrm{L})$ of each suspension were inoculated on Sabouraud dextrose agar and incubated for $48 \mathrm{~h}$ at $35^{\circ} \mathrm{C}$. Endpoints were determined by visual reading. Before reading, the microdilution trays were agitated and the MIC was computed as the lowest concentration of fluconazole to cause at least $50 \%$ inhibition of growth relative to that of the drug-free control. The trailing growth was considered when a reduced but persistent growth of the strain was detected in high concentrations of fluconazole.

Disk diffusion and E-test methods - The two methods were performed on Mueller-Hinton agar plates supplemented with $2 \%$ glucose and $0.5 \mathrm{mg}$ of methylene blue (GMB agar). For each isolate, two plates were inoculated by dipping two sterile cotton swabs into the standardised inoculum and evenly streaking the entire surface of the GMB agar in three directions, with a $60^{\circ}$ angle between them. After drying for $15 \mathrm{~min}$, one fluconazole E-test strip and one $25 \mu \mathrm{g}$ fluconazole disk (Cecon, Ltd, São Paulo, Brazil) were applied to the medium. The plates were incubated at $35^{\circ} \mathrm{C}$ and were read after $24 \mathrm{~h}$ and $48 \mathrm{~h}$. The MIC was read as the lowest concentrations producing an $80 \%$ reduction in growth. In the E-test, the values of MIC were read at the point where the ellipse of the inhibition zone intersected the strip. The residual growth of yeast strains from the boundary of the inhibition zone, pointing towards the E-test strip, was considered to be trailing growth. In the DT, the inhibition zone diameter was measured to the nearest $\mathrm{mm}$ at a point where there was a prominent reduction of growth $(80 \%)$. The CLSI inhibition zone diameter for fluconazole [susceptible ( $\geq 19 \mathrm{~mm})$, S-DD $(15-18 \mathrm{~mm})$ and resistant $(\leq 14 \mathrm{~mm})$ ] was used to categorise the disk diffusion results (NCCLS 2004). The yeast strains that showed a boundary of residual growth towards the disk of fluconazole were considered to exhibit trailing growth.

ATBF3 - An ATBF3 strip is composed of 32 wells that include a growth control and five antifungal drugs at different concentrations: 5-flucytosine from 4-16 $\mu \mathrm{g} / \mathrm{mL}(\log 2$ dilutions), amphotericin B from $0.5-16 \mu \mathrm{g} / \mathrm{mL}$, fluconazole 
from $1-128 \mu \mathrm{g} / \mathrm{mL}$, itraconazole from $0.125-4 \mu \mathrm{g} / \mathrm{mL}$ and voriconazole from $0.06-8 \mu \mathrm{g} / \mathrm{mL}$. The ATBF3 test was performed according to the manufacturer's instructions. The strips were read either visually or automatically by the mini Api ${ }^{\circledR}$ instrument (bioMérieux, Inc, France) at 24-48 $\mathrm{h}$ for Candida species. MICs for azoles and 5-flucytosine were determined according to the following criteria: no reduction or a slight reduction in turbidity (scores 4 and 3) was considered to be "no inhibition", while a prominent decrease to no growth (scores 2-0) corresponded to "inhibition". Only samples that remained optically clear were considered to be susceptible to amphotericin B (TorresRodríguez \& Alvarado-Ramírez 2007).

Vitek 2 system - Susceptibility testing with the Vitek 2 system (AST-YST card) was performed according to the manufacturer's instructions. From each strain, the standardised inoculum was placed in a Vitek 2 cassette, along with a sterile polystyrene test tube and a Vitek 2 card containing serial two-fold dilutions of fluconazole (1-64 $\mu \mathrm{g}$ / $\mathrm{mL})$ and voriconazole $(0.125-8 \mu \mathrm{g} / \mathrm{mL})$. After the loaded cassettes were placed in the Vitek 2 instrument, the cards were filled with the diluted yeast suspensions, incubated for $24 \mathrm{~h}$ and read automatically. The MIC results were interpreted as described by the M27-A3 guideline.

Flow cytometric assay - This method was performed as previously described (Ramani et al. 1997). Briefly, $0.1 \mathrm{~mL}$ aliquots of the standardised yeast suspension were mixed with $0.1 \mathrm{~mL}$ of serial two-fold dilutions of fluconazole in 12 by 75-mm tubes (Falcon 2054, Becton Dickinson, Lincoln Park, NJ, USA). The growth control received $0.1 \mathrm{~mL}$ of the drug diluent (RPMI-1640 medium) without fluconazole. All of the tubes were incubated without agitation at $35^{\circ} \mathrm{C}$ for $4 \mathrm{~h}$. Then, each dilution was shaken gently and $200 \mu \mathrm{L}$ of $25 \mathrm{mM}$ sodium desoxycholate and $4 \mu \mathrm{L}$ of $1 \%$ propidium iodide (Sigma) were added to each dilution, after which the tubes were carefully mixed again. Flow cytometry was performed on a FACS calibur cytometer (Becton Dickinson) on a sample volume of $75 \mu \mathrm{L}$ with a flow rate of $10 \mu \mathrm{L} / \mathrm{min}$. Cytometer parameters were as follows: forward scatter (linear gain 3.73), side scatter (gain 2.0), side scatter photomultiplier tubes $(270 \mathrm{~V})$ and $\log$ red fluorescence (FL2) $457 \mathrm{~V}$ log. Each flow cytometric susceptibility test analysed 10,000 yeast cells. MIC was computed as the lowest fluconazole concentration that resulted in a $50 \%$ increase in the mean channel fluorescence as compared to the growth control (Ramani et al. 1997, Ramani \& Chaturvedi 2000, Chaturvedi et al. 2004). The results were analysed using the WinMDI 2.8 program for fluorescence intensity and dispersion of microorganisms.

Analysis and interpretation of results - Both on-scale and off-scale results obtained by all the methods were included in the analysis. The off-scale MICs were converted to the next highest or lowest concentration. The MIC values were considered to be in essential agreement (EA) between the two methods when they were within two dilutions. Categorical agreement (CA) was assigned to Candida spp susceptibility testing results that fell within the same interpretive categories. Results were analysed based on the interpretive breakpoints for fluconazole [susceptible (S), $\leq 8 \mu \mathrm{g} / \mathrm{mL}$; susceptible dose dependent (SDD), $16-32 \mu \mathrm{g} / \mathrm{mL}$; resistant (R), $\geq 64 \mu \mathrm{g} / \mathrm{mL}]$. CA was assigned when both methods classified the susceptibilities of the isolates within the same interpretive categories (S, SDD or R). Discrepancies were considered "major" if an isolate classified as $\mathrm{S}$ by the reference method was classified as R by the commercial method and "very major" if an isolate classified as R by the reference method was classified as $\mathrm{S}$ by the commercial method. Errors were considered "minor" when there were discrepancies between the two methods in classifying SDD isolates as $\mathrm{S}$ or R or classifying $\mathrm{S}$ and $\mathrm{R}$ isolates as SDD.

Light and transmission electron microscopy analyses - In this set of experiments, the trailing positive $C$. tropicalis strain, 135, was used to investigate possible morphological alterations induced by fluconazole when compared to cells grown in its absence by both light microscopy and transmission electron microscopy.

For light microscopy analyses, aliquots of all dilutions of the BMD-N method, after a 24-h incubation, were placed on slides, stained by the Gram method and examined under a light microscope (Olympus, Japan) with a magnification of $1000 \mathrm{X}$. For ultrastructural analyses, untreated and fluconazole-treated cells were fixed in a solution containing $2.5 \%$ glutaraldehyde and $4 \%$ freshly prepared formaldehyde in a $100 \mathrm{mM}$ cacodylate buffer ( $\mathrm{pH}$ 7.2) for $1 \mathrm{~h}$ and washed three times in the same buffer. Then, the yeast strains were post-fixed in a $100 \mathrm{mM}$ cacodylate buffer $(7.2 \mathrm{pH})$ containing $1 \%$ osmium tetroxide, $0.8 \%$ potassium ferrocyanide and $5 \mathrm{mM}$ $\mathrm{CaCl}_{2}$ for $2 \mathrm{~h}$. After that, the yeast strains were dehydrated in an ethanol-graded series and critical point-dried with $\mathrm{CO}_{2}$. The specimens were coated with $3 \mathrm{~nm}$ of gold in a Balzers apparatus and ultrathin sections were observed in a Jeol 1200 EX transmission electron microscope at $80 \mathrm{kV}$ (Cunha et al. 2005).

Detection of Sap - Thirteen trailing-positive C. tropicalis strains were randomly chosen from among the 52 strains isolated in this work. The strains were grown in RPMI-1640 medium, with and without $128 \mu \mathrm{g} / \mathrm{mL}$ of fluconazole, for 24 and $48 \mathrm{~h}$ at $37^{\circ} \mathrm{C}$. All cultures were centrifuged to obtain concentrated cells, the yeast strains were washed in phosphate buffered saline (PBS) and a standardised inoculum $\left(5 \times 10^{6}\right.$ cells $)$ was prepared. The suspensions used for these experiments were fixed at $4^{\circ} \mathrm{C}$ in $4 \%$ paraformaldehyde in PBS ( $\mathrm{pH} 7.2$ ) for 20 min, followed by extensive washing in the same buffer. These fixed cells maintained their morphological integrity, as indicated by light microscope observation. They were incubated for $1 \mathrm{~h}$ at $25^{\circ} \mathrm{C}$ with a 1:250 dilution of rabbit anti-Sap1-3 antibody (kindly provided by Dr Nina Agabian, University of California, San Francisco, CA, USA) and then incubated for an additional hour with a 1:200 dilution of fluorescein-isothiocyanate-labelled goat anti-rabbit IgG. For flow cytometric analysis, these cells were examined in an EPICS ELITE flow cytometer (Coulter Electronics, USA) equipped with a $15-\mathrm{mW}$ argon laser emitting at $488 \mathrm{~nm}$. Untreated cells and those treated only with the anti-Sap and secondary antibody were used as controls. Each experimental population 
was then mapped by using a two-parameter histogram of forward-angle light scatter versus side scatter. The mapped population $(\mathrm{n}=10,000)$ was then analysed for $\log$ green fluorescence by using a single-parameter histogram (Braga-Silva et al. 2009).

\section{RESULTS AND DISCUSSION}

In this study, the majority of the 52 C. tropicalis strains were isolated from clinical specimens collected from invasive infections, mainly blood (55\%) and sterile fluids (24\%), such as ascite and pleural effusions. Whereas, strains from noninvasive lesions were mainly isolated from urine $(15 \%)$ and secretions $(6 \%)$. The major invasive potential of $C$. tropicalis in relation to other species of Candida, particularly C. albicans, has been previously reported (Colombo \& Guimarães 2003).

Susceptibility tests - We tested the susceptibility to fluconazole and the phenotypic expression of trailing growth for 52 C. tropicalis strains using the standard BMD-N method and six other methods for comparison (Table I). Data are shown as MIC ranges and the $\mathrm{MIC}_{50} \%$ and $\mathrm{MIC}_{90} \%$, except for the M44-A method, where the area of the inhibition zone $(\mathrm{mm})$ around the disk was considered.

The BMD-N method and most of the other methods could have a trailing effect so great as to make an isolate that appears susceptible after $24 \mathrm{~h}$ of incubation appear resistant after $48 \mathrm{~h}$. Therefore, it is recommended that the test readings be conducted at 24 and $48 \mathrm{~h}$ for the azoles to avoid the interference of this effect on the outcome of the test and to determine the category of susceptibility. In this work, the BMD-N and BMD-Ac methods and the E-test were read at 24 and $48 \mathrm{~h}$. ATBF3 was also examined after two incubation periods, but only the reading at $24 \mathrm{~h}$ was considered, while the flow cytometric, disk diffusion and Vitek 2 methods were read at $4 \mathrm{~h}, 24 \mathrm{~h}$ and up to $24 \mathrm{~h}$, respectively.

The ranges of the $24 \mathrm{~h}$-MIC results were similar among all methods. However, the readings were discrepant at $48 \mathrm{~h}$ compared to those at $24 \mathrm{~h}$, particularly for the BMD-N and ATBF3 methods. No significant differences were noted for the BMD-Ac and E-test methods. The discrepancies reflect the presence of the trailing end. $\mathrm{MIC}_{50} \%$ and $\mathrm{MIC}_{90} \%$ for fluconazole, as determined by six methods, were clearly concordant and ranged from $<1 \mu \mathrm{g} / \mathrm{mL}-2 \mu \mathrm{g} / \mathrm{mL}\left(\mathrm{MIC}_{50} \%\right)$ and $<$ $1-4 \mu \mathrm{g} / \mathrm{mL}\left(\mathrm{MIC}_{90} \%\right)$ (Table I).

The EA among all methods used for determining the MIC was excellent when compared with the BMD-N method, showing values of $98.1 \%$ for flow cytometry, $96.1 \%$ for Vitek 2 and ATBF3 and $92.3 \%$ for the BMD-Ac method. However, the E-test, which showed an EA of $80.8 \%$, was an exception. Thirteen discrepancies were noted between the MIC values of each strain for each method tested in relation to BMD-N, including 10 with the E-test, four with the BMD-Ac, two with the ATBF3 and two with the Vitek 2. Of the discrepancies noted, the MIC values obtained by the BMD-N were always lower than those obtained by the other methods. Similar EA results for testing various methods against the standard method excluding the E-test have been reported in the literature (Pfaller et al. 2007, Torres-Rodríguez \& Alvarado-Ramírez 2007, Cuenca-Estrella et al. 2010).

\section{TABLE I}

Susceptibility of 52 Candida tropicalis isolates to fluconazole and expression of trailing growth as determined by seven different methods

\begin{tabular}{|c|c|c|c|c|c|c|}
\hline \multirow[b]{2}{*}{ Method } & \multirow{2}{*}{$\begin{array}{l}\text { Incubation time } \\
\text { (h) }\end{array}$} & \multicolumn{3}{|c|}{$\operatorname{MIC}(\mu \mathrm{g} / \mathrm{mL})^{g}$} & \multirow{2}{*}{$\begin{array}{l}\text { Trailing }{ }^{h} \\
\qquad(\%)\end{array}$} & \multirow{2}{*}{$\begin{array}{l}\mathrm{EA}^{i} \\
(\%)\end{array}$} \\
\hline & & Range & $\mathrm{MIC}_{50} \%$ & $\mathrm{MIC}_{90} \%$ & & \\
\hline \multirow[t]{2}{*}{${\mathrm{BMD}-\mathrm{N}^{a}}$} & 24 & $0.125-2.0$ & 0.5 & 1.0 & - & - \\
\hline & 48 & $2.0-\geq 128$ & 0.5 & 2.0 & 98.08 & 100 \\
\hline \multirow[t]{2}{*}{$\mathrm{BMD}-\mathrm{Ac}^{a}$} & 24 & $0.25-4.0$ & 1.0 & 2.0 & - & - \\
\hline & 48 & $0.25-16.0$ & 1.0 & 2.0 & - & 92.31 \\
\hline \multirow[t]{2}{*}{$\mathrm{ATBF}^{b}$} & 24 & $\leq 1.0-16.0$ & $\leq 1.0$ & 2.0 & - & - \\
\hline & 48 & $\leq 1.0-\geq 128$ & - & - & 86.5 & 96.15 \\
\hline Vitek $2^{c}$ & 24 & $\leq 1.0-4.0$ & $\leq 1.0$ & $\leq 1.0$ & 100 & 96.15 \\
\hline Flow cytometry $^{d}$ & 4 & $0.125-2.0$ & 0.5 & 1.0 & 100 & 98.08 \\
\hline \multirow[t]{2}{*}{ E-test ${ }^{e}$} & 24 & $0.5-4.0$ & 1.5 & 3.0 & - & - \\
\hline & 48 & $1.0-6.0$ & 2.0 & 4.0 & 94.2 & 86.54 \\
\hline Disk diffusion M44-A $\mathrm{A}^{f}$ & 24 & $36-19$ & 27 & 21 & 96.15 & 100 \\
\hline
\end{tabular}

$a$ : standardised by Clinical and Laboratory Standards Institute [broth microdilution (BMD) tests in accordance with M27 A3 guideline at neutral pH (BMD-N) and acid pH (BMD-Ac)] (CLSI 2008); $b$ : ATB-Fungus 3 (ATBF3) (bioMérieux, Inc, France); $c$ : Vitek 2 (bioMérieux, Inc, France); $d$ : flow cytometry [as recommended by Ramani et al. (1997)]; e: E-test (AB Biodisk, Solna, Sweden); $f$ : standardised by National Committee on Clinical Laboratory Standards (NCCLS 2004) (size of inhibition zone in mm); $g$ : 50\% and $90 \%$ minimal inhibitory concentrations (MICs) at which $50 \%$ and $90 \%$ of the isolates tested are inhibited, respectively; $h$ : percentage of strains expressing trailing effect after $48 \mathrm{~h}$ of incubation in the presence of fluconazole; $i$ : EA: essential agreement. 
Previous studies also found similar EA results between E-test and BMD-N methods (Matar et al. 2003, CuencaEstrella et al. 2010). The results from the M44-A method were consistent with the categorisation of the strains as susceptible, in agreement with the other methods tested.

Despite of the discrepancies described, all methods identified all strains as susceptible to fluconazole, except for one strain that was SDD for the ATBF3 method and sensitive to other methods. Thus, although no major or very major error was defined, one minor error was defined. Table II shows the percentages of correlation between the different methods, in relation to EA. BMD-Ac and ATBF3 methods showed correlation values greater than $98 \%$ compared to three of the five other methods tested. Vitek 2 showed 100\% correlation with BMD-Ac and flow cytometry, which suggests less of an influence on reading due to trailing growth, which has also been shown by other authors (Ramani \& Chaturvedi 2000, Borghi et al. 2010). The lowest correlation rates were observed between flow cytometry and other methods, which is consistent with other previously reported results (Pfaller et al. 2007, Lee et al. 2009, Cuenca-Estrella et al. 2010). The presence of the trailing effect was assessed for all strains in relation to the seven methods tested (Table I). Percentages of the positivity of the strains ranging from $86.5-100 \%$ were detected for all methods, except for the BMD-Ac method, where trailing growth was not observed. This result agrees with the data of Marr et al. (1999). However, a previous study showed lower values for the presence of the trailing effect in C. tropicalis strains (Arthington-Skaggs et al. 2002). Ramani and Chaturvedi (2000) did not detect trailing growth in antifungal susceptibility testing performed using the flow cytometry method and suggested that the short incubation time was insufficient for the expression of this phenomenon. However, in our study we found a significant percentage of viable cells after $4 \mathrm{~h}$, even at high concentrations of the antifungal agent, and we considered these cells to represent trailing cells.
Morphological and ultrastructural changes - Several cellular and molecular mechanisms possibly responsible for trailing growth in Candida spp have been described (Lee et al. 2004, Vandeputte at al. 2005). Fluconazole, which is known to inhibit cell growth by disruption of sterol biosynthesis, increased cell size due to an apparently high porosity level of the cell wall. In consequence, these cells disintegrated, perhaps due to increased turgor pressure (Ramani \& Chaturvedi 2000). Herein, we used two different approaches to analyse the morphological alterations in C. tropicalis (strain 135) after treatment with fluconazole: (i) staining yeast cells using the Gram method (Fig. 1) and (ii) visualisation of general ultrastructural alterations by transmission electron microscopy (Fig. 2). Light microscopy revealed that in the presence of fluconazole $(8 \mu \mathrm{g} / \mathrm{mL}$ and $128 \mu \mathrm{g} / \mathrm{mL})$ several cells showed an altered morphology, such as increased size with greater dye uptake. Furthermore, the number of yeasts containing buds and cells showing incomplete cell division was increased after exposure to fluconazole compared to the fluconazole-untreated cells (Fig. 1). The increase in cell size had already been suggested by studies using flow cytometry (Ramani et al. 1997) and implies that the azoles may significantly affect the outer cell envelope (Belanger et al. 1997). The fluconazole-treated cells, even when grown at a higher concentration $(128 \mu \mathrm{g} / \mathrm{mL})$, were viable and able to grow on Sabouraud dextrose agar similarly to the control cells. Studies suggest that in the presence of fluconazole, $C$. albicans cells up-regulate the transcription of genes encoding the azole drug target, lanosterol demethylase (ERG 11), the target terbinafine, squalene epoxidase $(E R G I)$ or the azole and terbinafine efflux transporters (CDR1, CDR2 and MDR1) (Smith \& Edlind 2002). These same mechanisms and also point mutations in the ERG11 gene have been implicated as mechanisms of fluconazole resistance in C. albicans (Sanglard \& Odds 2002). Therefore, trailing isolates, although susceptible to fluconazole, are capable of expressing the same molecular mechanisms as SDD and R

TABLE II

Essential agreement rates between results given by six antifungal susceptibility tests

\begin{tabular}{|c|c|c|c|c|c|c|}
\hline \multirow[b]{2}{*}{ Methods } & \multicolumn{6}{|c|}{ Agreement $(\%)^{f}$} \\
\hline & BMD-N & BMD-Ac & Vitek 2 & E-test & ATBF3 & $\begin{array}{c}\text { Flow } \\
\text { cytometry }\end{array}$ \\
\hline $\mathrm{BMD}-\mathrm{N}^{a}$ & 100 & 92.3 & 96.1 & 80.8 & 96.1 & 98.1 \\
\hline $\mathrm{BMD}-\mathrm{Ac}^{a}$ & 92.3 & 100 & 100 & 100 & 98.1 & 88.5 \\
\hline Vitek $2^{b}$ & 96.1 & 100 & 100 & 96.1 & 98.1 & 100 \\
\hline E-test ${ }^{c}$ & 80.8 & 100 & 96.1 & 100 & 98.1 & 84.6 \\
\hline $\mathrm{ATBF}^{d}$ & 96.1 & 98.1 & 98.1 & 98.1 & 100 & 94.2 \\
\hline Flow cytometry ${ }^{e}$ & 98.1 & 88.5 & 100 & 84.6 & 94.2 & 100 \\
\hline
\end{tabular}

$a$ : standardised by Clinical and Laboratory Standards Institute [broth microdilution (BMD) tests in accordance with M27 A3 guideline at neutral pH (BMD-N) and acid pH (BMD-Ac)] (CLSI 2008); $b$ : Vitek 2 (bioMérieux, Inc, France); $c$ : E-test (AB Biodisk, Solna, Sweden); $d$ : ATB-Fungus 3 (ATBF3) (bioMérieux, Inc, France); $e$ : flow cytometry [as recommended by Ramani et al. (1997)]; $f:+/$ - one or two doubling concentrations. 
isolates in the presence of fluconazole, but regulate them differently (Lee et al. 2004). In C. tropicalis, azole resistance mechanisms have been studied less, but the overexpression of the CtERG11 gene (not the CtMDRI gene associated with a missense mutation in this gene) seemed to be responsible for the acquired azole resistance in a resistant clinical isolate of $C$. tropicalis (Vandeputte et al. 2005). However, the presence of trailing growth would have no greater significance in vivo because these strains were susceptible to the same concentrations of fluconazole used for susceptible strains (Arthington-Skaggs et al. 2000, Ostrosky-Zeichner et al. 2003).

The possible effect of trailing growth on ultrastructural changes in C. tropicalis was evaluated by transmission electron microscopy of yeast strains grown in concentrations of fluconazole above the MIC (Fig. 2). Electron micrographs of untreated $C$. tropicalis showed well-preserved yeast cells, presenting a compact cell wall (average thickness of $0.13 \mu \mathrm{m}$ ), a plasma membrane (with normal shape) and homogeneous cytoplasm (Fig. 2A and in the inset). However, yeast strains grown in the presence of $8 \mu \mathrm{g} / \mathrm{mL}$ of fluconazole and expressing trailing phenomenon showed important alterations, including mitochondrial swelling and a cell wall with an irregular shape and presenting wall thicknesses ranging from $0.14-0.28 \mu \mathrm{m}$ (Fig. 2B). The highest concentration of fluconazole $(128 \mu \mathrm{g} / \mathrm{mL})$ induced more pronounced changes in the

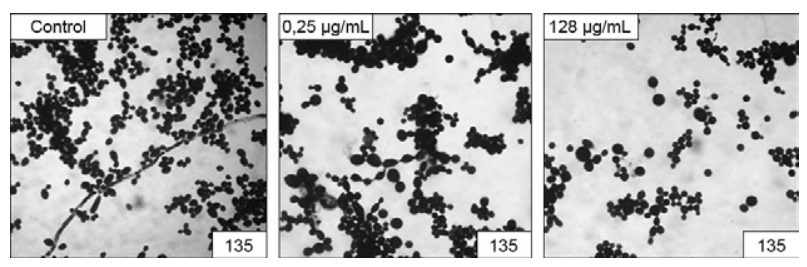

Fig. 1: smears of Candida tropicalis strain 135 grown in the absence (control) or in the presence of different concentrations of fluconazole. The slides were stained by Gram procedure and were examined under a light microscope with a magnification of 1000X.
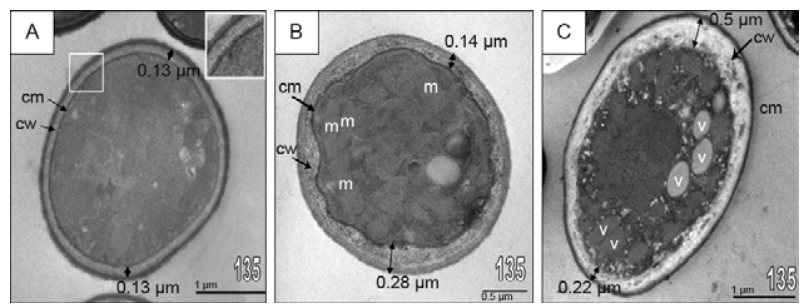

Fig. 2: electron micrographs showing the effect of fluconazole on the ultrastructure of Candida tropicalis strain 135. A: yeast cells grown in the absence of fluconazole presented a well-conserved morphology, with a distinct cell wall (cw) and preserved cytoplasmic membrane $(\mathrm{cm})$. The inset in shows a higher magnification of these two critical structures; B: yeasts cells exposed to fluconazole $(8 \mu \mathrm{g} / \mathrm{mL})$ presented irregular cytoplasmic membrane, distended cw and swollen of mitochondria (m); C: yeasts cells incubated with fluconazole at $128 \mu \mathrm{g} / \mathrm{mL}$ showed ruptures in the cytoplasmic membrane, $\mathrm{cw}$ enlargement and vacuolization (v). cytoplasm, such as the accumulation of several vacuoles and mitochondrial swelling. In addition, the cell wall showed an irregular shape and thicknesses ranging from $0.22-0.50 \mu \mathrm{m}$. Detachment and degradation of the cytoplasmic membrane was also evident (Fig. 2C). Previous works reported that Candida spp treated with azole agents or a synthetic arylquinucline derivate, exhibiting antifungal activity, presented similar ultrastructural alterations when examined under transmission electron microscopy (Belanger et al. 1997, Ishida et al. 2011).

Sap expression in trailing strains of C. tropicalis - A positive correlation between protease activity and antifungal susceptibility was already reported for C. albicans. In this sense, the $C$. albicans isolates from the human immunodeficiency virus-positive group that were characterised by higher levels of protease activity were also less susceptible to the widely used azole antifungal agents, ketoconazole and fluconazole (Ollert et al. 1995). C. albicans strains exposed to subinhibitory concentrations of antifungals belonging to the azole, echinocandin and pyrimidine analogue classes showed elevated activity of Sap2 in the culture filtrates (Wu et al. 2000, Ripeau et al. 2002). In addition, the expression of $S A P 2$ and activity of the secreted Sap 2 gene product was upregulated in fluconazole-exposed yeast cells (Copping et al. 2005). In fact, Navarathna et al. (2005) showed that the virulence of $C$. albicans strains increases upon exposure to subinhibitory concentrations of fluconazole in animal experiments. Recently, Mores et al. (2011) described that biofilms of $C$. albicans challenged by subMICs of fluconazole tend to secrete higher quantities of Sap compared to non-treated cells.

\section{TABLE III}

Expression of cell-associated aspartic proteases by Candida tropicalis strains as determined by flow cytometric assay ${ }^{a}$

\begin{tabular}{lcccc}
\hline & \multicolumn{2}{c}{ Fluorescence $^{b}(\%)$} & & Rate \\
\cline { 2 - 3 } Strains & $24 \mathrm{~h}$ & $48 \mathrm{~h}$ & & $48 / 24$ \\
\hline 57 & $44.30 \pm 3.68$ & $21.33 \pm 2.60$ & 0.48 \\
135 & $13.20 \pm 1.27$ & $9.65 \pm 0.31$ & 0.73 \\
152 & $10.65 \pm 0.97$ & $11.66 \pm 0.21$ & 1.09 \\
153 & $1.67 \pm 0.01$ & $8.40 \pm 0.10$ & 5.03 \\
252 & $11.53 \pm 0.11$ & $18.37 \pm 1.17$ & 1.59 \\
257 & $9.85 \pm 0.35$ & $22.42 \pm 0.29$ & 2.28 \\
264 & $8.03 \pm 0.01$ & $30.67 \pm 2.12$ & 3.82 \\
277 & $4.09 \pm 0.05$ & $15.87 \pm 0.91$ & 3.88 \\
750 & $19.54 \pm 0.26$ & $37.55 \pm 3.18$ & 1.92 \\
1048 & $8.30 \pm 0.01$ & $10.77 \pm 0.59$ & 1.30 \\
1053 & $7.03 \pm 0.65$ & $4.33 \pm 0.21$ & 0.62 \\
1063 & $14.06 \pm 1.48$ & $8.82 \pm 1.37$ & 0.63 \\
1067 & $26.70 \pm 0.29$ & $36.20 \pm 4.55$ & 1.36 \\
\hline
\end{tabular}

$a$ : according to Braga-Silva et al. (2009); $b$ : $24 \mathrm{~h}$ and $48 \mathrm{~h}$ incubation time of the culture. 
With this task in mind, we assessed the production of Saps in 13 C. tropicalis strains, expressing trailing growth, through flow cytometry. The fluorescence intensity obtained with cells grown in the presence of fluconazole for $24 \mathrm{~h}$ or $48 \mathrm{~h}$ was compared to that obtained with control cells (cultured in the absence of antifungal) after staining with antibodies raised against Sap1-3 from C. albicans (Table III). Our results showed that all the C. tropicalis strains after exposure to fluconazole up-regulated on surface Sap expression, in relation to untreated yeast strains. In this context, yeast strains treated with fluconazole for $24 \mathrm{~h}$ had levels of surface Saps 1.67-44.3-fold higher than control cells $(\mathrm{p}<0.05)$. Similarly, yeast strains under fluconazole pressure for $48 \mathrm{~h}$ presented 4.33-37.55-fold higher Sap levels in relation to untreated yeast strains $(\mathrm{p}<0.05)$ (Table II). In addition, the majority of the strains $(69.2 \%)$ showed an increased expression of Saps after $48 \mathrm{~h}$ of exposure to fluconazole when compared to a $24 \mathrm{~h}$ exposure. The significance of these findings in relation to $C$. tropicalis virulence in the presence of fluconazole in vivo is not clear, but experiments in vivo with $C$. albicans showed that Sap production may contribute to the pathogenesis of inflammatory mucosal lesions in a model of vaginal candidiasis (De Bernardis et al. 1999). Thus, the role of Saps of C. tropicalis in the pathogenicity of invasive or superficial infections caused by this species needs to be investigated. We demonstrated that even in the presence of high concentrations of fluconazole, $C$. tropicalis still produced significant levels of Saps, suggesting a possible role in the virulence characteristics of this species.

Taken together, our results show that $C$. tropicalis strains isolated from invasive and noninvasive infections were susceptible to fluconazole and all methods of antifungal susceptibility tested showed similar performance. Moreover, the strains of $C$. tropicalis had a high level of trailing growth and this phenomenon may have an influence on biological and structural properties of yeast cells. However, despite these physiological and structural alterations, several cells within the population maintained their viability and were able to multiply and produce, which are factors possibly involved in the pathogenesis of $C$. tropicalis.

\section{REFERENCES}

Anaissie EJ, Paetznick VL, Ensign LG, Espinel-Ingroff A, Galgiani JN, Hitchcock CA, Larocco M, Patterson T, Pfaller MA, Rex JH, Rinaldi MG 1996. Microdilution antifungal susceptibility testing of Candida albicans and Cryptococcus neoformans with and without agitation: an eight-center collaborative study. Antimicrob Agents Chemother 40: 2387-2391.

Arthington-Skaggs BA, Lee-Yong W, Ciblak MA, Frade JP, Brandt ME, Hajjeh RE, Harrison LE, Sofair AN, Warnock DM 2002. Comparison of visual and spectrophotometric methods of broth microdilution MIC endpoint determination and evaluation of sterol quantitation method for in vitro susceptibility testing of fluconazole and itraconazole against trailing and non-trailing Candida isolates. Antimicrob Agents Chemother 46: 2477-2481.

Arthington-Skaggs BA, Warnock DW, Morrison CJ 2000. Quantitation of Candida albicans ergosterol content improves the correlation between in vitro antifungal susceptibility test results and in vivo outcome after fluconazole treatment in a murine model of invasive candidiasis. Antimicrob Agents Chemother 44: 2081-2085.
Belanger P, Nast CC, Fratti R, Sanati H, Ghannoum M 1997. Voriconazole (UK-109,496) inhibits the growth and alters the morphology of fluconazole-susceptible and -resistant Candida species. Antimicrob Agents Chemother 41: 1840-1842.

Borghi E, Iatta R, Sciotta R, Biassoni C, Cuna T, Montagna MT, Morace G 2010. Comparative evaluation of the Vitek 2 yeast susceptibility test and CLSI broth microdilution reference method for testing antifungal susceptibility of invasive fungal isolates in Italy: the Gisia 3 Study. J Clin Microbiol 48: 3153-3157.

Braga-Silva LA, Mesquita DGA, Ribeiro MD, Carvalho SMF, Fracalanzza SEL, Santos ALS 2009. Trailing end-point phenotype antibiotic-sensitive strains of Candida albicans produce different amounts of aspartyl peptidases. Braz J Med Biol Res 42: 765770.

Braga-Silva LA, Santos ALS 2011. Aspartic protease inhibitors as potential anti-Candida albicans drugs: impacts on fungal biology, virulence and pathogenesis. Curr Med Chem 18: 2401-2419.

Cantón E, Viudes A, Pemán J 2001. Infección sistémica nosocomial por levaduras. Rev Iberoam Micol 18: 51-55.

Chaturvedi V, Ramani R, Pfaller MA 2004. Collaborative study of the NCCLS and flow cytometry methods for antifungal susceptibility testing of Candida albicans. J Clin Microbiol 42: 2249-2251.

CLSI - Clinical Laboratory Standards Institute 2008. Reference method for broth dilution antifungal susceptibility testing of yeasts. M27-A3. CLSI 28: 1-30.

Colombo AL, Guimarães T 2003. Epidemiologia das infecções hematogênicas por Candida spp. Rev Soc Bras Med Trop 36: 599-607.

Copping VM, Barelle CJ, Hube B, Gow NA, Brown AJ, Odds FC 2005. Exposure of Candida albicans to antifungal agents affects expression of SAP2 and SAP9 secreted proteinase genes. $J$ Antimicrob Chemother 55: 645-654.

Cuenca-Estrella M, Gomez-Lopez A, Alastruey-Izquierdo A, BernalMartinez L, Cuesta I, Buitrago MJ, Rodriguez-Tudela JI 2010. Comparison of the Vitek 2 antifungal susceptibility system with the Clinical and Laboratory Standards Institute (CLSI) and European Committee on Antimicrobial Susceptibility Testing (EUCAST), Broth Microdilution Reference Methods and with the Sensititre Yeast One and Etest Techniques for in vitro detection of antifungal resistance in yeast isolates. J Clin Microbiol 48: 1782-1786.

Cunha MML, Franzen AJ, Alviano DS, Zanardi E, Alviano CS, Souza W, Rozental S 2005. Inhibition of melanin synthesis pathway by tricyclazole increases susceptibility of Fonsecaea pedrosoi against mouse macrophages. Microsc Res Tech 68: 377-384.

De Bernardis F, Arancia S, Morelli L, Hube B, Sanglard D, Schafer W 1999. Evidence that members of the secretory aspartyl proteinase gene family, in particular $S A P 2$, are virulence factors for Candida vaginitis. J Infect Dis 179: 201-208.

Fridkin SK, Kaufman D, Edwards JR, Shetty S, Horan T 2006. Changing incidence of Candida bloodstream infections among NICU patients in the United States. Pediatrics 117: 1680-1687.

Ishida K, Rodrigues JCF, Carmmerer S, Urbiba J, Gilbert I, de Souza W, Rozental S 2011. Synthetic arylquinuclidine derivates exhibit antifungal activity against Candida albicans, Candida tropicalis and Candida parapsilosis. Ann Clin Microbiol Antimicrob 10: 3.

Lee MK, Willians LE, Warnock DW, Arthington-Skaggs BA 2004. Drug resistance genes and trailing growth in Candida albicans isolates. J Antimicrob Chemother 53: 217-224.

Lee SC, Lo HJ, Fung CP, Lee N, See LC 2009. Disk diffusion test and E-test with enriched Mueller-Hinton agar for determining susceptibility of Candida species to voriconazole and fluconazole. J Microbiol Immunol Infect 42: 148-153. 
Marr KA, Rustad TG, Rex JH, White TC 1999. The trailing end point phenotype in antifungal susceptibility testing is $\mathrm{pH}$ dependent. Antimicrob Agents Chemother 43: 1383-1386.

Matar MJ, Ostrosky-Zeichner L, Pretznick VL, Rodriguez JR, Chan EJ, Rex JH 2003. Correlation between E-test, disk diffusion and microdilution methods for antifungal susceptibility testing of fluconazole and voriconazole. Antimicrob Agents Chemother 47: $1647-1651$.

Mimica LMJ, Ueda SMY, Martino MDV, Navarini A, Martini LJ 2009. Diagnóstico de infecção por Candida: avaliação de testes de identificação de espécies e caracterização do perfil de suscetibilidade. J Bras Patol Med Lab 45: 17-23.

Mores AU, Souza RD, Cavalca L, de Paula e Carvalho A, Gursky LC, Rosa RT, Samaranayake LP, Rosa EA 2011. Enhancement of secretory aspartyl protease production in biofilms of Candida albicans exposed to sub-inhibitory concentrations of fluconazole. Mycoses 54: 195-201.

Naglik JR, Challacombe SJ, Hube B 2003. Candida albicans secreted aspartyl proteinases in virulence and pathogenesis. Microbiol Mol Biol Rev 67: 400-428.

Navarathna DH, Hornby JM, Hoerrmann N, Parkhurst AM, Duhamel GE, Nickerson KW 2005. Enhanced pathogenicity of Candida albicans pre-treated with subinhibitory concentrations of fluconazole in a mouse model of disseminated candidiasis. $J$ Antimicrob Chemother 56: 1156-1159.

NCCLS/CLSI - National Committee for Clinical Laboratory Standards/Clinical Laboratory Standards Institute 2004. Methods for antifungal disk diffusion susceptibility testing of yeasts: approved guideline, M44-A. Available from: clsi.org/source/orders/ free/m44-a2.pdf

Odds FC 1985. Candida albicans proteinase as a virulence factor in the pathogenesis of Candida infections. Zentralbl Bakteriol Microbiol Hyg [A] 260: 539-542.

Ollert MW, Wende C, Gorlich M, McMullan-Vogel CG, Borg-von Zepelin M, Vogel CW, Korting HC 1995. Increased expression of Candida albicans secretory proteinase, a putative virulence factor in isolates from human immunodeficiency virus-positive patients. J Clin Microbiol 33: 2543-2549.

Ostrosky-Zeichner L, Rex JH, Pappas PG, Hamill RJ, Larsen RA, Horowitz HW, Powderly WG, Hyslop N, Kauffman CA, Cleary J, Mangino JE, Lee J 2003. Antifungal susceptibility survey of 2,000 bloodstreams Candida isolates in the United States. Antimicrob Agents Chemother 47: 3149-3154.

Pfaller MA, Diekema DJ 2004. Twelve years of fluconazole in clinical practice: global trends in species distribution and fluconazole susceptibility of bloodstream isolates of Candida. Clin Microbiol Infect 10: 11-23.

Pfaller MA, Diekema DJ 2007. Epidemiology of invasive candidiasis: persistent public health problem. Clin Microbiol Rev 20: 133-163.

Pfaller MA, Diekema DJ, Procop DG, Rinaldi MG 2007. Multicenter comparison of the Vitek 2 yeast susceptibility test with the CLSI broth microdilution reference method for testing fluconazole against Candida spp. J Clin Microbiol 45: 796-802.

Ramani R, Chaturvedi V 2000. Flow cytometry antifungal susceptibility testing of pathogenic yeasts other than Candida albicans and comparison with the NCCLS broth microdilution test. Antimicrob Agents Chemother 44: 2752-2758.

Ramani R, Ramani A, Wong SJ 1997. Rapid flow cytometric susceptibility testing of Candida albicans. J Clin Microbiol 35: 2320-2324.

Revankar SG, Kirkpatrick WR, Matee RK, Fothergill AW, Redding SW, Rinaldi SG, Patterson TF 1998. Interpretation of trailing endpoints in antifungal susceptibility testing by the National Committee for Clinical Laboratory Standards method. J Clin Microbiol 36: 153-156.

Ripeau JS, Aumont F, Belhumeur P, Ostrosky-Zeichner L, Rex JH, de Repentigny L 2002. Effect of the echinocandin caspofungin on expression of Candida albicans secretory aspartyl proteinases and phospholipase in vitro. Antimicrob Agents Chemother 46: 3096-3100.

Rodriguez-Tudela JL, Berenguer J, Martinez-Suarez JV, Sanchez R 1996. Comparison of a spectrophotometric microdilution method with RPMI-2\% glucose with the National Committee for Clinical Laboratory Standards reference macrodilution method M27-P for in vitro susceptibility testing of amphotericin B, flucytosine and fluconazol against Candida albicans. Antimicrob Agents Chemoth 40: 1998-2003.

Ruan SY, Hsueh PR 2009. Invasive candidiasis: an overview from Taiwan. J Formos Med Assoc 108: 443-451.

Sandven P 2000. Epidemiology of candidemia. Rev Iberoam Micol 17: $73-81$.

Sanglard D, Odds FC 2002. Resistance of Candida species to antifungal agents: molecular mechanisms and clinical consequences. Lancet Infect Dis 2: 73-85.

Smith WL, Edlind TD 2002. Histone deacetylase inhibitors enhance Candida albicans sensitivity to azoles and related antifungals: correlation with reduction in $C D R$ and $E R G$ upregulation. Antimicrob Agents Chemother 46: 3532-3539.

Tornatore MA, Noskin GA, Hacek DM, Obias AA, Peterson LRL 1997. Effects of incubation time and buffer concentration on in vitro activities of antifungal agents against Candida albicans. J Clin Microbiol 35: 1473-1476.

Torres-Rodríguez J, Alvarado-Ramírez E 2007. In vitro susceptibilities to yeast using the ATB Fungus 2 method compared with Sensititre Yeast One and standard CLSI (NCCLS) M27-A2 methods. $J$ Antimicrob Chemother 60: 658-661.

Vandeputte P, Larcher G, Berges T, Renier G, Chabasse D, Bouchara JP 2005. Mechanisms of azole resistance in a clinical isolate of Candida tropicalis. Antimicrob Agents Chemother 49: 4608-4615.

Warnock DW 2007. Trends in the epidemiology of invasive fungal infections. Jpn J Med Mycol 48: 1-12.

Wu T, Wright K, Hurst SF, Morrison CJ 2000. Enhanced extracellular production of aspartyl proteinase, a virulence factor, by Candida albicans isolates following growth in subinhibitory concentrations of fluconazole. Antimicrob Agents Chemother 44: $1200-1208$

Zaugg C, Borg-von Zepelin M, Reichard U, Sanglard D, Monod M 2001. Secreted aspartic proteinase family of Candida tropicalis. Infect Immun 69: 405-412. 